\title{
ENERGY SCATTERING FOR HARTREE EQUATIONS
}

\author{
KENJI NAKANISHI
}

\begin{abstract}
We give an alternative proof for the result in [7] that the scattering operators are well-defined in the whole energy space for a class of Hatree equations. Our proof allows potentials which are flat at the origin. Moreover, our proof gives the desired global a priori space-time estimates.
\end{abstract}

\section{Introduction}

In this note, we study the scattering theory in the energy space for Hartree type equations:

$$
i \dot{u}-\Delta u+\left(V *|u|^{2}\right) u=0,
$$

where $u=u(t, x),(t, x) \in \mathbb{R}^{1+n}$, with $n \geq 3, V=V(x)$, and $\dot{u}=\partial u / \partial t$. In [7, Proposition 4.3], it was shown that the wave operators and the scattering operator are well-defined homeomorphisms in the energy space $H^{1}\left(\mathbb{R}^{n}\right)$ if the following conditions on $V$ are fulfilled:

$$
V \in L^{p_{1}}+L^{p_{2}}
$$

for some $p_{1}, p_{2} \in(\max (1, n / 4), n / 2)$, and $V(x)=v(|x|)$ for some nonnegative nonincreasing function $v$ satisfying

$$
v\left(r_{1}\right)-v\left(r_{2}\right) \geq C\left(r_{1}^{\alpha}-r_{2}^{\alpha}\right) \quad \text { for } 0<r_{1}<r_{2} \leq a,
$$

for some $C, a, \alpha>0$. The scattering theory has been studied also in some weighted spaces $[4,9,8,12]$. The scattering in the energy space has been studied also in the case of the pointwise nonlinearity:

$$
i \dot{u}-\Delta u+p(u)=0,
$$

where $p$ is a complex valued function. See $[5,6,2]$.

In this note, we give another proof for the main part of the above result in [7], namely the asymptotic completeness for (1.1) in $H^{1}$. We do not need the unnatural assumption (1.3) (cf. [7, Remark 4.1]). Thus, we can deal with potentials $V$ which are flat around the origin, for example:

$$
V(x)= \begin{cases}1, & |x|<1 \\ 0, & |x|>1\end{cases}
$$

Received December 14, 1998.

This author's research is supported by Research Fellowships of the Japan Society for the Promotion of Science for Young Scientists. 
Moreover, our proof gives the desired global a priori space-time estimates in terms of the energy, which were not obtained in [7] (cf. [7, Remark 4.2]). In the case of (1.4), such an estimate was obtained in [2]. Our arguments are similar to those in [11] and [10], and even simpler. The main idea is a nice combination of Morawetz-type estimates and propagation estimates (see Section 5), which may be regarded as 'multiplicative' combination, whereas the argument in [7] may be regarded as 'additive' combination. There is a similar argument in [2].

We can exclude the assumption (1.3) because we use new estimates of Morawetz type (Lemma 3.1), which are sharper versions of the estimate derived in [11], and a certain related Sobolev-type inequality (Lemma 3.3). The usual Morawetz estimates exploit only the properties of $\Delta$, but the new estimates exploit the properties of $i \partial_{t}-\Delta$, so that they yield more information on the solutions peculiar to nonlinear Schrödinger equations. Such an estimate for nonlinear wave equations was derived in [10, Proposition 4.4]. The main result of this note is the following.

Theorem 1.1. Assume that $V \in L^{p_{1}}+L^{p_{2}}$ with some $p_{1}, p_{2} \geq 1$ satisfying $n / 2<p_{1} \leq p_{2}<n / 4$. Assume that $V(x)=v(|x|)$ for some nonnegative nonincreasing $v$. Then, the wave operators and the scattering operator for (1.1) are homeomorphisms in $H^{1}\left(\mathbb{R}^{n}\right)$. Precisely, for any solution $u$ of (1.1) with $u(0) \in H^{1}\left(\mathbb{R}^{n}\right)$, there exists a solution $w$ of the free Schrödinger equation

$$
i \dot{w}-\Delta w=0,
$$

with $w(0) \in H^{1}\left(\mathbb{R}^{n}\right)$ such that $\|u(t)-w(t)\|_{H^{1}} \rightarrow 0$ as $t \rightarrow \infty$. Moreover, the correspondence $u(0) \mapsto w(0)$ defines a homeomorphism in $H^{1}\left(\mathbb{R}^{n}\right)$ (we have the same result for $t \rightarrow-\infty)$.

The arguments in this note can be used also to show the asymptotic completeness for (1.4) under the conditions on the function $p$ :

$$
\begin{gathered}
p(0)=0, \quad|p(u)-p(v)| \leq C|u-v| \min \left((|u|+|v|)^{q_{1}},(|u|+|v|)^{q_{2}}\right), \\
\text { for some } q_{1}, q_{2} \in(1+4 / n, 1+4 /(n-2)), \\
{ }^{\exists} P: \mathbb{C} \rightarrow \mathbb{R}, \text { s.t. } \partial_{\bar{z}} P(z)=p(z), \quad P(0)=0, \\
G(u):=\Re(\bar{u} p(u))-P(u) \geq 0,
\end{gathered}
$$

which are essentially weaker than those needed in $[5,6,2]$, with respect to (1.7).

Throughout this note, we always assume (1.2) for fixed $p_{1}$ and $p_{2}$ satisfying

$$
p_{1}=\frac{n}{2+2 \varepsilon}, \quad p_{2}=\max \left(\frac{n}{4-2 \varepsilon}, 1\right)
$$

for some $\varepsilon>0$ small. We fix this $\varepsilon \in(0,1 / 4)$ also.

The rest of this paper is organized as follows. In the next section we give several notations and basic estimates. In Section 3, we derive some new variants of the Morawetz estimate. In Section 4, we prove a weighted global estimate for 
the space-time norm. In Section 5, we prove the desired global estimate for the space-time norm, from which Theorem 1.1 easily follows.

\section{Notations and basic estimates}

In this section, we introduce several notations and basic estimates. As usual, we denote by $C$ auxiliary positive constants, and sometimes write as $C(a, b, \ldots)$ to indicate that the constant depends only on $a, b, \ldots$ and that the dependence is continuous (we will use this convention for constants which are not denoted by ' $C$ '). We fix $n$ and $\varepsilon$ in (1.8) and ignore the dependence of constants on $n, \varepsilon$. We denote by $B_{q, r}^{\sigma}$ the usual inhomogeneous Besov spaces (see, e.g., [1]).

Now we will define the space-time norms used in this note. We will sometimes abbreviate them as 'ST-norms'.

$$
\begin{array}{ll}
(\widetilde{B} ; I):=L^{\infty}\left(I ; B_{\infty, 2}^{1-n / 2}\left(\mathbb{R}^{n}\right)\right), & (B ; I):=L^{\infty}\left(I ; B_{\infty, \infty}^{1-n / 2-2 \varepsilon}\left(\mathbb{R}^{n}\right)\right), \\
(\widetilde{X} ; I):=L^{6}\left(I ; B_{6 n /(3 n-2), 2}^{1}\left(\mathbb{R}^{n}\right)\right), & (X ; I):=L^{6}\left(I ; B_{6 n /(3 n-2-6 \varepsilon), 2}^{1-2 \varepsilon}\left(\mathbb{R}^{n}\right)\right), \\
(K ; I):=L^{3}\left(I ; B_{6 n /(3 n-4), 2}^{1}\left(\mathbb{R}^{n}\right)\right), & (\bar{K} ; I):=L^{3 / 2}\left(I ; B_{6 n /(3 n+4), 2}^{1}\left(\mathbb{R}^{n}\right)\right) .
\end{array}
$$

We will sometimes omit the interval $I$ in (2.1). We fix a smooth cut-off function $h$ satisfying

$$
h \in C^{\infty}(\mathbb{R}), \quad 0 \leq h \leq 1, \quad h(t)= \begin{cases}1, & t \geq 1 \\ 0, & t \leq 0 .\end{cases}
$$

Denote by $\mathcal{F} \varphi=\widetilde{\varphi}$ the Fourier transform of $\varphi$ and define the Littlewood-Paley dyadic decomposition:

$$
\begin{aligned}
& \psi_{j}:=\mathcal{F}^{-1} h\left(2-2^{-j}|\xi|\right) \in \mathcal{S}\left(\mathbb{R}^{n}\right), \quad \psi_{j}^{C}:=\delta-\psi_{j}, \\
& \varphi_{j}:= \begin{cases}\psi_{j}-\psi_{j-1}, & \text { for } j \in \mathbb{N}, \\
\psi_{0}, & \text { for } j=0 .\end{cases}
\end{aligned}
$$

Denote

$$
\begin{gathered}
f(u):=\left(V *|u|^{2}\right) u, \quad F(u):=\left(V *|u|^{2}\right)|u|^{2}, \\
E(u):=\int_{\mathbb{R}^{n}}|u|^{2}+|\nabla u|^{2}+F(u) d x,
\end{gathered}
$$

where $\nabla u=\left(\partial u / \partial x_{1}, \ldots, \partial u / \partial x_{n}\right) . E(u)$ is a conserved quantity for (1.1). The integral equation associated to (1.1) can be written as:

$$
u(t)=e^{-i \Delta t} u(0)+i \int_{0}^{t} e^{-i \Delta(t-s)} f(u(s)) d s .
$$


Now we collect several basic estimates on the space-time norms. By the Sobolev embedding, we have for any $j \in \mathbb{N}$,

$$
\begin{gathered}
\|u\|_{(B)} \leq C\|u\|_{(\widetilde{B})} \leq C\|u\|_{L_{t}^{\infty}\left(H^{1}\right)}, \\
\left\|\varphi_{j} * u\right\|_{(B)}+\left\|\psi_{j}^{C} * u\right\|_{(B)} \leq C 2^{-2 \varepsilon j}\|u\|_{(\widetilde{B})}, \\
\|u\|_{(X)} \leq C\|u\|_{(\widetilde{X})}, \\
\left\|\varphi_{j} * u\right\|_{(X)}+\left\|\psi_{j}^{C} * u\right\|_{(X)} \leq C 2^{-\varepsilon j}\|u\|_{(\widetilde{X})} .
\end{gathered}
$$

By the Sobolev embedding and Hölder's and Young's inequalities, we have

$$
\begin{aligned}
\|f(u)\|_{(\bar{K})} & \leq C\|u\|_{(K)}\|u\|_{L_{t}^{6}\left(L^{\left.q_{1} \cap L^{q_{2}}\right)}\right.}^{2}\|V\|_{L^{p_{1}}+L^{p_{2}}} \\
& \leq C\|u\|_{(K)}\|u\|_{(X)}^{2},
\end{aligned}
$$

where $q_{1}:=6 n /(3 n-2-6 \varepsilon)$ and $q_{2}:=\min (6 n /(3 n-8+6 \varepsilon), 3 n / 2)$. By the complex interpolation and the Sobolev embedding, we have

$$
\|u\|_{(X)} \leq C\|u\|_{(K)}^{1 / 2}\|u\|_{L_{t}^{\infty}\left(H^{1}\right)}^{1 / 2-2 \varepsilon / n}\|u\|_{(B)}^{2 \varepsilon / n} .
$$

By the Strichartz estimate, we have for any $t>0$,

$$
\|u(t)\|_{H^{1}}+\|u\|_{(K ;(0, t))}+\|u\|_{(\widetilde{X} ;(0, t))} \leq C\|u(0)\|_{H^{1}}+C\|i \dot{u}-\Delta u\|_{(\bar{K} ;(0, t))} .
$$

Using the above estimates, it is easy to prove the unique global existence of the solution and existence of the wave operators defined everywhere in $H^{1}\left(\mathbb{R}^{n}\right)$. See [7] for more detail. By the above estimates, we have the following lemma. The idea is essentially due to Bourgain [3].

Lemma 2.1. Let $u$ satisfy (1.1) on an interval $I$ with $E(u)=E<\infty$ and $\|u\|_{(X ; I)}=\eta$. There exists a constant $\eta_{0}(E) \in(0,1)$ such that if $\eta \leq \eta_{0}(E)$ we have a subinterval $J \subset I, R>0$ and $c \in \mathbb{R}^{n}$ satisfying $|J| \geq C(E, \eta)$, $R \leq C(E, \eta)$ and

$$
\int_{|x-c|<R} \min \left(|u(t)|,|u(t)|^{s}\right) d x \geq C(E, \eta, s),
$$

for any $t \in J$ and any $s \geq 1$.

Proof. This is almost the same as [11, Lemma 4.1], so we give a rather sketchy proof. By $(2.9)$ and $(2.7)$, if $\eta_{0}(E)$ is sufficiently small, it follows that $\|u\|_{(K ; I)}<$ $C(E)$ and $\|u\|_{(\widetilde{X} ; I)}<C(E)$ from $\|u\|_{(X ; I)} \leq \eta_{0}$. Then, from (2.8), we have $\|u\|_{(B ; I)}>C(E, \eta)$. This means that we have some $T \in I, c \in \mathbb{R}^{n}$ and $j \in \mathbb{N} \cup\{0\}$ such that

$$
\left|2^{(1-n / 2-2 \varepsilon) j} \varphi_{j} * u(T, c)\right| \geq C(E, \eta) .
$$


By (2.6), we have $j \leq C(E, \eta)$. Moreover, there exists some $k<C(E, \eta)$ such that

$$
\frac{\eta}{2} \leq\left\|\psi_{k} * u\right\|_{(X ; I)} .
$$

On the other hand, by the Sobolev embedding and Hölder's inequality, we have

$$
\left\|\psi_{k} * u\right\|_{(X ; I)} \leq|I|^{1 / 6} C(k)\|u\|_{L^{\infty}\left(I ; H^{1}\right)} \leq C(E, \eta)|I|^{1 / 6} .
$$

So we have $|I| \geq C(E, \eta)$. From the equation and the Sobolev embedding, we have

$$
\left\|\varphi_{j} *(u(t)-u(T))\right\|_{L^{\infty}} \leq C(j)\|u(t)-u(T)\|_{H^{-1}} \leq C(E, \eta)|t-T| .
$$

Thus, there is some interval $J \subset I$ such that $|J| \geq C(E, \eta)$ and that we have (2.11) for any $T \in J$. From (2.11) and Young's inequality, we have

$$
\|u(T)\|_{L^{1}(|x-c|<R)} \geq C(E, \eta),
$$

for some large $R<C(E, \eta)$. Then, by Hölder's inequality we obtain the desired result.

\section{Morawetz-type estimates}

In this section, we derive certain variants of the Morawetz estimates with space-time weights. They are sharper versions of the estimate in [11, Lemma 5.2 ], where it was derived in the case of the pointwise nonlinearity.

Lemma 3.1. Assume that $V(x)=v(|x|)$ for some nonnegative nonincreasing $v$. Let $u$ be a global solution of (1.1) with $E(u)=E<\infty$. Then we have for any $0<\nu \leq 1$,

$$
\iint_{\mathbb{R}^{1+n}} \frac{|2 t \nabla u+i x u|^{2}}{|t|^{1-\nu}(|t|+|x|)^{2+\nu}} d x d t \leq C(E, \nu)
$$

Proof. We will use the following notations.

$$
\begin{gathered}
r=|x|, \quad \theta=\frac{x}{r}, \quad \lambda=\sqrt{t^{2}+r^{2}}, \quad \gamma=\frac{r}{t}, \\
u_{r}=\theta \cdot \nabla u, \quad u_{\theta}=\nabla u-\theta u_{r} .
\end{gathered}
$$

It suffices to consider the estimate for $t>0$. We give only a formal proof assuming that $u$ is sufficiently smooth. It can be easily extended to general finite energy solutions. We will use the following multiplier:

$$
m=2 \varphi(\gamma) u_{r}+g(\gamma) \frac{u}{t}+i \psi(\gamma) u
$$

where

$$
\varphi(\gamma)=\int_{0}^{\gamma} \frac{d s}{\langle s\rangle^{2+\nu}}, \quad \psi(\gamma)=\int_{\infty}^{\gamma} \frac{s d s}{\langle s\rangle^{2+\nu}}, \quad g=\varphi^{\prime}+\frac{n-1}{\gamma} \varphi
$$


where $\langle s\rangle=\sqrt{1+s^{2}}$. In the case $\nu=1, m$ is identical with $m_{p}$ in [11, (5.18)]. Then we have $\psi^{\prime}=\gamma \varphi^{\prime}$, and $\varphi^{\prime}$ is nonnegative and nonincreasing. In particular we have $\varphi / \gamma \geq \varphi^{\prime}$. In a way similar to $[11,(5.19),(5.20)]$, we have

$$
\begin{aligned}
\Re\{(i \dot{u}-\Delta u) \bar{m}\}=\partial_{t}\left\{\varphi(\gamma) \Im\left(u \overline{u_{r}}\right)+\frac{|u|^{2}}{2} \psi(\gamma)\right\} & \\
+\nabla & \Re\left\{-\nabla u \overline{m_{p}}+\varphi(\gamma)\left\{\Im(u \bar{u})+|\nabla u|^{2}\right\}+\frac{|u|^{2}}{2 t^{2}} g^{\prime}(\gamma) \theta\right\} \\
+ & \frac{\varphi^{\prime}(\gamma)}{2 t^{3}}|2 t \nabla u+i x u|^{2}+2\left(\frac{\varphi(\gamma)}{\gamma}-\varphi^{\prime}(\gamma)\right) \frac{\left|u_{\theta}\right|^{2}}{t} \\
& -\frac{|u|^{2}}{2 t^{3}}\left(\partial_{\gamma}^{2}+\frac{n-1}{\gamma} \partial_{\gamma}\right) g(\gamma) .
\end{aligned}
$$

The last term is further calculated as

$$
\begin{aligned}
& \left(\partial_{\gamma}^{2}+\frac{n-1}{\gamma} \partial_{\gamma}\right) g(\gamma) \\
& \quad=\left(\partial_{\gamma}^{2}+2 \frac{n-1}{\gamma} \partial_{\gamma}\right) \varphi^{\prime}(\gamma)+\frac{(n-1)(n-3)}{\gamma^{2}}\left(\varphi^{\prime}(\gamma)-\frac{\varphi(\gamma)}{\gamma}\right)
\end{aligned}
$$

The last term is nonpositive, and the first term in the right hand side is explicitly computated as

$$
\left(\partial_{\gamma}^{2}+2 \frac{n-1}{\gamma} \partial_{\gamma}\right) \varphi^{\prime}(\gamma)=-(2+\nu)\left\{\frac{2 n-5-\nu}{\langle\gamma\rangle^{4+\nu}}+\frac{\nu+4}{\langle\gamma\rangle^{6+\nu}}\right\} \leq 0
$$

Thus, the last three terms in (3.5) are all nonnegative. So, integrating (3.5) over $[S, T] \times \mathbb{R}^{n}$, we obtain

$$
\begin{aligned}
{\left[\int_{\mathbb{R}^{n}}-\varphi(\gamma) \Im\left(u \overline{u_{r}}\right)-\frac{|u|^{2}}{2} \psi(\gamma) d x\right]_{S}^{T} \geq \int_{S}^{T} } & \int_{\mathbb{R}^{n}} \frac{\varphi^{\prime}(\gamma)}{2 t^{3}}|2 t \nabla u+i x u|^{2} d x d t \\
& +\int_{S}^{T} \int_{\mathbb{R}^{n}} \Re\{f(u) \bar{m}\} d x d t
\end{aligned}
$$

Since the left hand side is bounded by the energy, it suffices to show that the last term is nonnegative. Let $h(x):=\varphi(\gamma) \theta$. Denote by $(\cdot, \cdot)$ the inner product in $L^{2}\left(\mathbb{R}^{n}\right)$. Then we have

$$
\begin{aligned}
\Re(f(u), m) & =\left(V *|u|^{2}, \Re\{2 \bar{u} h \cdot \nabla u\}+|u|^{2} \nabla \cdot h\right) \\
& =\left(V *|u|^{2}, \nabla \cdot\left(h|u|^{2}\right)\right)=-\left(\nabla V *|u|^{2}, h|u|^{2}\right) .
\end{aligned}
$$


Then, we have

$$
\begin{aligned}
& \left(\nabla V *|u|^{2}, h|u|^{2}\right)=\iint v^{\prime}(|x-y|) \frac{x-y}{|x-y|} \cdot h(x)|u|^{2}(x)|u|^{2}(y) d x d y \\
& =\frac{1}{2} \iint v^{\prime}(|x-y|) \frac{x-y}{|x-y|} \cdot(h(x)-h(y))|u|^{2}(x)|u|^{2}(y) d x d y
\end{aligned}
$$

where in the second identity, we used the antisymmetry of the second member for $h(x) \leftrightarrow h(y)$. Since $v^{\prime} \leq 0$, it suffices to show that

$$
(x-y) \cdot(h(x)-h(y)) \geq 0,
$$

which is equivalent to that $\nabla h$ is everywhere nonnegative definite. Since we have

$$
\nabla h=\frac{1}{t}\left\{\varphi^{\prime}(\gamma)^{t} \theta \theta+\frac{\varphi(\gamma)}{\gamma}\left(I-{ }^{t} \theta \theta\right)\right\},
$$

and $\varphi / \gamma \geq \varphi^{\prime}>0$, so $\nabla h$ is actually positive definite. Thus we obtain the desired result.

Remark 3.2. There are still sharper estimates. For example, choosing $\varphi^{\prime}=$ $\langle\gamma\rangle(\log (2+\gamma))^{-2}$ in $(3.4)$, we obtain

$$
\iint \frac{|2 t \nabla u+i x u|^{2}}{|t|\left(|t|^{2}+|x|^{2}\right)(\log (2+|x / t|))^{2}} d x d t \leq C(E, \eta) .
$$

On the other hand, for any nontrivial solution $u$, we have

$$
\iint \frac{|2 t \nabla u+i x u|^{2}}{|t|\left(|t|^{2}+|x|^{2}\right)} d x d t=\infty
$$

which can be easily seen by

$$
\lim _{t \rightarrow 0} \int \frac{|2 t \nabla u+i x u|^{2}}{\left(|t|^{2}+|x|^{2}\right)} d x=\int|u|^{2} d x .
$$

To use the above Morawetz-type estimate, we use the following weighted Sobolev-type inequality.

Lemma 3.3. Let $n \geq 3$ and $\frac{n-1}{n-2} \leq p<n$. Let $w(r)$ be a nonnegative function which is absolutely continuous locally on $(0, \infty)$ and for some $\mu \in(0,1)$,

$$
\frac{-w^{\prime} r}{n w} \leq \mu \text { for a.e.r. }
$$

Then for any smooth function $\varphi(x)$ and any real-valued measurable function $T(x)$, we have

$$
\int_{\mathbb{R}^{n}}|\varphi|^{p^{*}} w d x \leq \frac{C}{(1-\mu)^{p}}\|\nabla \varphi\|_{L^{p}}^{p^{*}-p} \int_{\mathbb{R}^{n}}|\nabla \varphi+i x T \varphi|^{p} w d x
$$

where $p^{*}:=n p /(n-p) . C>0$ depends only on $n$ and $p$. 
Proof. Denote $\varphi_{T}=\nabla \varphi+i x T \varphi$. Using the obvious identities:

$$
\begin{gathered}
\Re\left(\varphi \overline{\varphi_{r}}\right)=\Re\left(\varphi \theta \cdot \overline{\varphi_{T}}\right), \\
\varphi_{\theta}=\varphi_{T}-\theta\left(\theta \cdot \varphi_{T}\right),
\end{gathered}
$$

we obtain in the same way as in [10, Lemma 3.8],

$$
\int_{\mathbb{R}^{n}}|\varphi|^{p^{*}} w d x \leq \frac{C}{(1-\mu)^{p}}\left\|r^{\nu} \varphi\right\|_{L_{r}^{\infty} L_{\theta}^{(n-1) / \nu}}^{p^{*}-p} \int_{\mathbb{R}^{n}}|\nabla \varphi+i x T \varphi|^{p} w d x
$$

where $\nu=(n-p) / p$, and the norm of $L_{r}^{\infty} L_{\theta}^{(n-1) / \nu}$ is the supremum of $\|\varphi(r \cdot)\|_{L^{(n-1) / \nu\left(S^{n-1}\right)}}$ for all $r>0$. Then, by [10, Proposition 3.7], we have

$$
\left\|r^{\nu} \varphi\right\|_{L_{r}^{\infty} L_{\theta}^{(n-1) / \nu}} \leq C\|\nabla \varphi\|_{L^{p}}
$$

so we obtain the desired result.

Remark 3.4. In the same way, we can improve (3.20) as

$$
\left\|r^{\nu} \varphi\right\|_{L_{\theta}^{(n-1) / \nu} L_{r}^{\infty}} \leq C\|\nabla \varphi+i x T \varphi\|_{L^{p}}
$$

where $T=T(x)$ is any real-valued measurable function. See the proof of $[10$, Proposition 3.7]. Then, the above lemma is improved as

$$
\int_{\mathbb{R}^{n}}|\varphi|^{p^{*}} w d x \leq \frac{C}{(1-\mu)^{p}}\|\nabla \varphi+i x S \varphi\|_{L^{p}}^{p^{*}-p} \int_{\mathbb{R}^{n}}|\nabla \varphi+i x T \varphi|^{p} w d x
$$

where $S=S(x)$ and $T=T(x)$ are any real-valued measurable functions. Taking $w=1$, we have in particular,

$$
\|\varphi\|_{L^{p^{*}}} \leq C\|\nabla \varphi+i x T \varphi\|_{L^{p}} .
$$

From the above two lemmas, we obtain the following a priori estimate.

Corollary 3.5. Assume that $V(x)=v(|x|)$ for some nonnegative nonincreasing $v$. Let $u$ be a global solution of (1.1) with $E(u)=E<\infty$. Then we have for any $\nu>0$,

$$
\iint_{\mathbb{R}^{1+n}} \frac{|t|^{1+\nu}|u|^{2^{*}}}{(|t|+|x|)^{2+\nu}} d x d t \leq C(E, \nu)
$$

where $2^{*}=2 n /(n-2)$. Remark that the right hand side does not depend on $V$.

Proof. It is easily checked that $w=(|t|+|x|)^{-\alpha}$ satisfies the condition (3.16) with some $\mu \in(0,1)$ independent of $t$, if $0<\alpha<n$. Remark that (3.24) becomes weaker as $\nu$ becomes larger. 


\section{Weighted global estimate for ST-norms}

Using Corollary 3.5 and an estimate for the propagation, we obtain the following lemma, which means a global estimate on ST-norms with $1 / t$-weight.

Lemma 4.1. Assume that $V(x)=v(|x|)$ for some nonnegative nonincreasing $v$. Let $u$ be a global solution of (1.1) with $E(u)=E<\infty$. Let $N \in \mathbb{N}$, $0=T_{0}<\cdots<T_{N}, I_{j}=\left(T_{j-1}, T_{j}\right)$, and $\|u\|_{\left(X ; I_{j}\right)}=\eta \in\left(0, \eta_{0}(E)\right)$ for any $j$ ( $\eta_{0}$ is as in Lemma 2.1). Then, there exists $t_{j} \in I_{j}$ for each $j$ such that

$$
\sum_{j=1}^{N} \frac{1}{\left(t_{j}+1\right)} \leq C(E, \eta)
$$

Proof. Since this is essentially the same as [11, Lemma 6.1], we give only the outline of the proof. We combine the Morawetz type estimate (3.24) with the approximate finite propagation property: For any closed $B \subset \mathbb{R}^{n}$, we have

$$
\int_{B(R)}|u(T)|^{2} d x \geq \int_{B}|u(0)|^{2} d x-C_{1}(E) T / R,
$$

for any $T, R>0$ and some $C_{1}$, where $B(R):=\left\{x \in \mathbb{R}^{n}|\exists y \in B| x-y \mid, \leq R\right\}$. (4.2) can be easily proved in the same way as in [11, Lemma 6.2]. By Lemma 2.1, we have, for each $j, R<C(E, \eta), c_{j} \in \mathbb{R}^{n}$ and $J_{j} \subset I_{j}$ with $\left|J_{j}\right|>C(E, \eta)$, such that

$$
\int_{\left|x-c_{j}\right|<R} \min \left(|u(t)|^{2^{*}},|u(t)|^{2}\right) d x>C_{2}(E, \eta),
$$

for any $t \in J_{j}$ and some $C_{2}$. Now let $t_{j}=\inf J_{j}$. Let $M=M(E, \eta)$ be so large that $C_{1}(E) / M<C_{2}(E, \eta) / 8$. Then we consider a family of fat cones $K_{k}=\left\{(t, x)|| x-X_{k}|<M| t-T_{k} \mid+3 R, t \geq T_{k}\right\}$ centered at $\left(T_{k}, X_{k}\right)$ such that each $B_{j}:=\left\{\left(t_{j}, x\right)|| x-c_{j} \mid<R\right\}$ is included in some $K_{k}$, that any $D_{k}:=\left\{\left(T_{k}, x\right)|| x-X_{k} \mid<R\right\}$ is identical with some $B_{j}$ and that $D_{k}$ does not intersect with the other fat cones. Then, by (4.2), the number of the fat cones $\left\{K_{k}\right\}$ is estimated by the total charge $\|u\|_{L_{x}^{2}}^{2}$ and $C_{2}(E, \eta)$. Now we consider (3.24) with its center at each $\left(T_{k}, X_{k}\right)$ and sum them up for all $k$ :

$$
\sum_{k} \iint_{K_{k}} \frac{|u|^{2^{*}}}{\left|t-T_{k}\right|+R} d x d t<C(E, M) \#\left\{K_{k}\right\}<C(E, \eta) .
$$

Since all $B_{j}$ are covered by $\left\{K_{k}\right\}$, we obtain the desired result from this and (4.3). 


\section{Global space-time estimate}

To obtain the scattering result, it suffices to show that for any finite energy solution, certain space-time norms are globally bounded. So, the following is the main result of this note.

Proposition 5.1. Assume that $V(x)=v(|x|)$ for some nonnegative nonincreasing $v$. Let $u$ be a global solution of (1.1) with finite energy $E(u)=E<\infty$. Then we have $\|u\|_{(X ; \mathbb{R})} \leq C(E)$.

Proof. Let $N \in \mathbb{N}, 0=T_{0}<T_{1}<\cdots<T_{N}, I_{j}=\left(T_{j-1}, T_{j}\right)$ and $\|u\|_{\left(X ; I_{j}\right)}=\eta$ where $\eta \in\left(0, \eta_{0}(E) / 2\right)$ will be determined later depending on $E$. $\quad\left(\eta_{0}\right.$ is as in Lemma 2.1.) By Lemma 4.1, we have

$$
\sum_{j=1}^{N} \frac{1}{T_{j}+1} \leq C(E, \eta) .
$$

Since $1 / t$ is not integrable on $(0, \infty)$, for any $L>0$ there exists $N_{0}(L, E, \eta) \in \mathbb{N}$ such that if $N>N_{0}$, then we have some $j<N-1$ such that $\left|I_{j}\right|>L$. So, repeating this argument, and applying (5.1) with suitably shifted time origin, we deduce that for any $L>0$ and any $M \in \mathbb{N}$ there exists $N_{1}(L, M, E, \eta)=$ $M N_{0}(L, E, \eta)$ such that if $N>N_{1}$, then we have $M$ distinct indices $j<N-1$ such that $\left|I_{j}\right|>L$ (we will determine $L$ and $M$ later so large depending on $E$ and $\eta$ ). Suppose $N>N_{1}$ and denote by $S$ the totality of such indices $\{j\}$. Let $u_{0}$ be the solution of (1.6) with the same initial data as $u$. By the Strichartz estimate (2.9), we have

$$
\sum_{j \in S}\left\|u_{0}\right\|_{\left(X ; I_{j}\right)}^{6} \leq\left\|u_{0}\right\|_{(X ;(0, \infty))}^{6} \leq C\left\|u_{0}(0)\right\|_{H^{1}}^{6} \leq C_{1}(E)^{6},
$$

for some $C_{1}(E)>0$. So, there exists some $j \in S$ satisfying

$$
\left\|u_{0}\right\|_{\left(X ; I_{j}\right)} \leq C_{1}(E) / M^{\frac{1}{6}}
$$

Now we set $M=M(E, \eta)$ so large that the right hand side becomes smaller than $\eta / 8$. From (2.5), we have for $t \in I_{j+1}$,

$$
u(t)=u_{0}(t)+i \int_{0}^{t} e^{-i \Delta(t-s)} f(u(s)) d s .
$$

We split the integral into those on $\left(0, T_{j-1}\right)$ and $\left(T_{j-1}, t\right)$, and denote them by $\mathcal{I}_{1}$ and $\mathcal{I}_{2}$, respectively. Using the decay property of the linear Schrödinger equation, Hölder's and Young's inequalities and the Sobolev embedding, we have

$$
\begin{aligned}
\left\|e^{-i \Delta t} f(\varphi)\right\|_{L^{2 n /(n-3)}} & \leq C|t|^{-3 / 2}\|f(\varphi)\|_{L^{2 n /(n+3)}} \\
& \leq C|t|^{-3 / 2}\|V\|_{L^{p_{1}}+L^{p_{2}}}\|\varphi\|_{L^{q_{1} \cap L^{q_{2}}}}^{3} \\
& \leq C|t|^{-3 / 2}\|\varphi\|_{H^{1}}^{3},
\end{aligned}
$$


where $q_{1}=6 n /(3 n-1-4 \varepsilon)$ and $q_{2}=\min (6 n /(3 n-5+4 \varepsilon), 6 n /(n+3))$. Using the Sobolev embedding, we have

$$
\begin{aligned}
\left\|\mathcal{I}_{1}\right\|_{\left(\widetilde{B} ; I_{j+1}\right)} \leq C\left\|\mathcal{I}_{1}\right\|_{L^{\infty}\left(I_{j+1} ; L^{2 n /(n-3)}\right)} & \\
& \leq C(E) \int_{0}^{T_{j-1}}\left|T_{j}-s\right|^{-3 / 2} d s \leq C(E) L^{-1 / 2} .
\end{aligned}
$$

As in the proof of Lemma 2.1, we have $\|u\|_{\left(K ; I_{j} \cup I_{j+1}\right)}<C(E)$ from $\|u\|_{\left(X ; I_{j} \cup I_{j+1}\right)}<\eta_{0}(E)$. Then, by (2.9) and (2.7), we have

$$
\begin{aligned}
& \left\|\mathcal{I}_{2}\right\|_{L^{\infty}\left(I_{j+1} ; H^{1}\right)}+\left\|\mathcal{I}_{2}\right\|_{\left(K ; I_{j+1}\right)}+\left\|\mathcal{I}_{2}\right\|_{\left(X ; I_{j+1}\right)} \\
& \quad \leq C\|u\|_{\left(K ; I_{j} \cup I_{j+1}\right)}\|u\|_{\left(X ; I_{j} \cup I_{j+1}\right)}^{2} \leq C_{2}(E) \eta^{2}
\end{aligned}
$$

for some $C_{2}(E)>0$. Now we set $\eta=\eta(E)>0$ so small that $C_{2}(E) \eta^{2}<\eta / 8$. By (2.8), (5.7) and (5.6), we have

$$
\left\|\mathcal{I}_{1}\right\|_{\left(X ; I_{j+1}\right)} \leq C(E)\left\|\mathcal{I}_{1}\right\|_{\left(B ; I_{j+1}\right)}^{2 \varepsilon / n} \leq C_{3}(E) L^{-\varepsilon / n}
$$

for some $C_{3}(E)>0$. Now we set $L=L(E, \eta)$ so large that $C_{3}(E) L^{-\varepsilon / n}<\eta / 8$. Then, from (5.3), (5.8) and (5.7), we have

$$
\|u\|_{\left(X ; I_{j+1}\right)} \leq C_{1}(E) / M^{\frac{1}{6}}+C_{3}(E) L^{-\varepsilon / n}+C_{2}(E) \eta^{2}<3 \eta / 8,
$$

This is a contradiction. So, for such small $\eta=\eta(E)$, large $M=M(E, \eta)$ and large $L=L(E, \eta)$, we can not have $N>N_{1}(L, M, E, \eta)$. Then we obtain the desired bound

$$
\|u\|_{(X ;(0, \infty))} \leq C\left(N_{1}\right) \eta \leq C(E) .
$$

From this estimate, we can easily obtain the scattering result Theorem 1.1. See [7].

\section{Acknowledgments}

I am grateful to Professor Yoshio Tsutsumi for his valuable advices and encouragement for this work. I also thank the referee for his comments, which clarified this paper.

\section{References}

[1] J. Bergh and J. Löfström, Interpolation spaces, An introduction. Grundlehren der Mathematischen Wissenschaften, No. 223., Springer-Verlag, Berlin-New York, 1976.

[2] J. Bourgain, Scattering in the energy space and below for $3 D$ NLS, J. Anal. Math. 75 (1998), 267-297.

[3] J. Bourgain, Global wellposedness of defocusing critical nonlinear Schrödinger equation in the radial case, J. Amer. Math. Soc. 12 (1999), 145-171.

[4] J. Ginibre and G. Velo, On a class of nonlinear Schrödinger equations with nonlocal interaction, Math. Z. 170 (1980), 109-136. 
[5] J. Ginibre and G. Velo, Scattering theory in the energy space for a class of non-linear Schrödinger equations, J. Math. Pures Appl. (9) 64 (1985), no. 4, 363-401.

[6] J. Ginibre and G. Velo, Time decay of finite energy solutions of the non linear KleinGordon and Schrödinger equations, Ann. Inst. H. Poincaré Phys. Théor. 43 (1985), 399442.

[7] J. Ginibre and G. Velo, Scattering theory in the energy space for a class of Hartree equations, preprint.

[8] N. Hayashi and T. Ozawa, Scattering theory in the weighted $L^{2}\left(R^{n}\right)$ spaces for some Schrödinger equations, Ann. Inst. H. Poincaré Phys. Théor. 48 (1988), 17-37.

[9] N. Hayashi and Y. Tsutsumi, Scattering theory for Hartree type equations, Ann. Inst. H. Poincaré Phys. Théor. 46 (1987), 187-213.

[10] K. Nakanishi, Unique global existence and asymptotic behaviour of solutions for wave equations with non-coercive critical nonlinearity, to appear in Comm. Partial Differential Equations.

[10] K. Nakanishi, Scattering theory for nonlinear Klein-Gordon equation with Sobolev critical power, Internat. Math. Res. Notices (1999), 31-60.

[11] K. Nakanishi, Energy scattering for nonlinear Klein-Gordon and Schrödinger equations in spatial dimensions 1 and 2 , preprint.

[12] H. Nawa and T. Ozawa, Nonlinear scattering with nonlocal interaction, Comm. Math. Phys. 146 (1992), 259-275.

Graduate School of Mathematical Sciences, University of Tokyo, 3-8-1 Komaba, Meguro, Tokyo 153-8914, JAPAN

E-mail address: nakanisi@ms.u-tokyo.ac.jp 